hep-th/9803194

MRI-PHY/P980338

\title{
Stable Non-BPS States in String Theory
}

\author{
Ashoke Sen f \\ Mehta Research Institute of Mathematics \\ and Mathematical Physics \\ Chhatnag Road, Jhoosi, Allahabad 211019, INDIA
}

\begin{abstract}
Many string theories contain states which are not BPS, but are stable due to charge conservation. In many cases the description of these states in the strong coupling limit remains unknown despite the existence of a weakly coupled dual theory. However, we show that in some cases duality symmetries in string theory do enable us to identify these states in the strong coupling limit and calculate their masses. We also speculate that in some of the other cases the missing states might arise from non-supersymmetric analog of D0-branes.
\end{abstract}

\footnotetext{
${ }^{1}$ E-mail: sen@mri.ernet.in
} 


\section{Contents}

\begin{tabular}{lll}
\hline 1 & Introduction & 2
\end{tabular}

2 Dirichlet 6-brane on Top of an Orientifold 6-plane 4

\begin{tabular}{|lll|}
\hline 3 & Dirichlet 7-brane on Top of an Orientifold 7-plane & 8
\end{tabular}

4 Dirichlet 4-brane on Top of an Orientifold 4-plane 10

\begin{tabular}{|lll}
5 & Other D $p$-brane $-\mathrm{O} p$-plane Systems & 12
\end{tabular}

6 Some Speculations about the Missing States 13

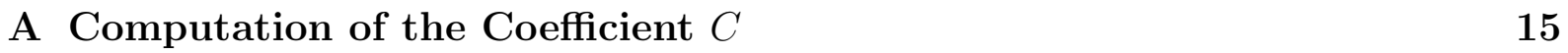

\section{Introduction}

During the last few years significant progress has been made towards understanding the strong coupling dynamics of string theories using duality symmetries which relate a strongly coupled string theory to a weakly coupled string/supergravity theory. However, despite this progress, some of the simple questions concerning the strongly coupled string theory remain unanswered in many cases. One such question will be the topic of discussion of this paper.

Spectra of string theories often contain a special class of states known as BPS states[四]. The mass of a BPS state is completely determined by its charge. Furthermore, BPS states are stable, and the degeneracy of a BPS state is independent of all continuous parameters of the theory, including the coupling constant. Due to these properties, the spectrum of BPS states in a string theory can, in principle, be reliably calculated at all values of the coupling by analyzing the theory at weak coupling. However, quite often a string theory also contains states which are stable but not BPS. The stability of these states follows from simple argument involving charge conservation; they are the lighest states carrying a given set of charges, and hence cannot decay. The masses of these states at weak coupling can be calculated reliably using string perturbation theory. The question that we shall be interested in is: what happens to these states at strong coupling? In this case there is no analog of the BPS formula that gives a relation between the mass and the charge at all values of the coupling; and calculating the masses of these states at strong coupling 
requires an explicit study of the strong coupling dynamics of the theory. The question of degeneracy of these states is easier to settle; generically we would expect that the lightest states carrying a given charge will belong to a single supermultiplet, since any accidental degeneracy of these states at the string tree level will be lifted by quantum corrections.

The most familiar example of this kind is seen in the $\operatorname{Spin}(32) / Z_{2}$ heterotic string theory[2] - the states in the spinor representation of the gauge group are stable due to charge conservation but are not BPS states. In the fermionic formulation of the heterotic string theory these states are obtained by putting periodic boundary condition on all the left-moving fermions, and have mass of the order of the string scale. As we increase the string coupling constant, the masses of these states will get renormalized, but the lightest states transforming in the spinor representation of $\operatorname{Spin}(32) / Z_{2}$ will remain stable. Let us denote the mass of such a state by:

$$
\left(\alpha^{\prime}\right)^{-1 / 2} f\left(g_{S}\right)
$$

where $\left(2 \pi \alpha^{\prime}\right)^{-1}$ is the string tension, $g_{S}$ is the string couping constant, and $f\left(g_{S}\right)$ is some function of $g_{S}$ that can be calculated in string perturbation theory. The question we are interested in is: how does $f\left(g_{S}\right)$ behave for large $g_{S}$ ?

Since for large $g_{S}$ this theory can be described by weakly coupled type I string theory[阿], one might hope that the answer can be found by analysing this weakly coupled theory. Unfortunately our understanding of the solitonic spectrum of weakly coupled type I string theory is not sufficiently complete so as to enable us to answer this question. Clearly the states we are looking for should correspond to a non-supersymmetric soliton of type I theory belonging to the spinor representation of the gauge group. At a qualitative level one might even describe these as arising from small loops of D-strings [4, 5, 6]. However such a description does not enable us to calculate the precise mass of these states. In section 6 we speculate on the possible origin of these states from non-supersymmetric D0-branes in type I string theory.

There are however examples in string theory where strong-weak coupling duality does help us determine the spectrum of stable non-BPS states in the strong coupling limit. These examples involve a Dirichlet $p$-brane (D $p$-brane) [7] of type II string theory on top of an orientifold $p$-plane (Op-plane) [8, 9] with SO projection. The world-volume theory of this configuration has an $\mathrm{SO}(2)$ gauge field originating from open strings with both ends lying on the $\mathrm{D} p$-brane. Open string states which originate on the $\mathrm{D} p$-brane, goes once 
around the $\mathrm{O} p$-plane, and ends on the $\mathrm{D} p$-brane are charged under this $\mathrm{SO}(2)$ gauge group. We shall normalize the gauge fields so that this state carries unit $\mathrm{SO}(2)$ charge. When the $\mathrm{D} p$-brane coincides with the $\mathrm{O} p$-plane, the classical mass of this state vanishes. However, the supersymmetric ground state of this system is projected out by the orientifolding operation [9], and hence in the weak coupling limit the lowest mass state in this sector has mass of the order of the string scale coming from the string oscillator contribution. Although this state is not a BPS state, it is stable under quantum corrections, being the lowest mass state carrying charge under the $\mathrm{SO}(2)$ gauge group. Let us denote the quantum corrected mass of the lightest charged state in the world-volume theory by

$$
\left(\alpha^{\prime}\right)^{-1 / 2} f_{p}\left(g_{S}\right)
$$

$f_{p}\left(g_{S}\right)$ is calculable in string perturbation theory, but we shall be interested in knowing the behaviour of $f_{p}\left(g_{S}\right)$ for large $g_{S}$. W We shall show that at least in two cases, $p=6$ and $p=7, f_{p}\left(g_{S}\right)$ can be completely determined in the strong coupling limit with the help of duality symmetries, although for $p=7$ the meaning of the strong coupling limit becomes somewhat subtle. For $p=4$ the behaviour of $f_{p}\left(g_{S}\right)$ for large $g_{S}$ can be determined up to an overall multiplicative numerical factor. Finally for $p=3$ and $p=5$, the $\mathrm{D} p$-brane - Op-plane configuration in the strong coupling limit can be mapped to appropriate configurations of orbifold / orientifold planes and D-branes in the dual weakly coupled type IIB string theory. Unfortunately, the precise identification of the lighest states carrying $\mathrm{SO}(2)$ charge is not known in these dual descriptions. Thus the situation in these two cases is analogous to the situation with spinor states in type I string theory. In section 6 we speculate on the possible identification of these states. 3

\section{Dirichlet 6-brane on Top of an Orientifold 6-plane}

This case was discussed earlier in [11], but we include it here for completeness. The theory under consideration is type IIA string theory. The orientifold 6-plane is given by:

$$
R^{6,1} \times\left(R^{3} /(-1)^{F_{L}} \cdot \Omega \cdot \mathcal{I}_{3}\right),
$$

\footnotetext{
${ }^{2}$ Although in this case we are analyzing a state in the world-volume theory of a brane rather than in the string theory itself, we can easily regarded this as a state in a compactified string theory by taking the directions transverse to the brane / plane to be compact but large, with other $\mathrm{D} p$-branes and $\mathrm{O} p$-planes situated sufficiently far away.

${ }^{3}$ Extremal but non-BPS black holes of the kind analyzed in ref. 10 might provide other examples of this kind in some specific region of the moduli space.
} 
where $R^{6,1}$ is the $(6+1)$ dimensional Minkowski space, $R^{3}$ is the three dimensional Euclidean space, $\mathcal{I}_{3}$ denotes a transformation that reverses the sign of all the coordinates of $R^{3}, F_{L}$ denotes the contribution to the space-time fermion number from the left-moving sector of the world-sheet and $\Omega$ denotes the world-sheet parity transformation. In the strong coupling limit the type IIA string theory is described by M-theory / eleven dimensional supergravity theory compactified on a circle of large radius $R$, and the coincident D6-brane - O6-plane system is described by the double cover $\overline{\mathcal{N}}$ of the Atiyah-Hitchin space 12, 13] in M-theory [14, 15, 11]. The eleven dimensional metric $g_{M N}^{(11)}$ takes the form:

$$
d s_{11}^{2} \equiv \sum_{M, N=0}^{10} g_{M N}^{(11)} d x^{M} d x^{N}=\sum_{\mu, \nu=0}^{6} \eta_{\mu \nu} d x^{\mu} d x^{\nu}+\frac{R^{2}}{4} d s_{A H}^{2},
$$

where $\eta_{\mu \nu}$ is the $(6+1)$ dimensional Minkowski metric, and

$$
d s_{A H}^{2}=f(\rho)^{2} d \rho^{2}+a(\rho)^{2} \sigma_{1}^{2}+b(\rho)^{2} \sigma_{2}^{2}+c(\rho)^{2} \sigma_{3}^{2},
$$

where,

$$
\begin{aligned}
\sigma_{1} & =-\sin \psi d \theta+\cos \psi \sin \theta d \phi, \\
\sigma_{2} & =\cos \psi d \theta+\sin \psi \sin \theta d \phi, \\
\sigma_{3} & =d \psi+\cos \theta d \phi,
\end{aligned}
$$

$x^{0}, \ldots x^{7}, \rho, \theta, \phi, \psi$ denote the coordinates of the eleven dimensional space, and $f, a, b$ and $c$ are known functions of $\rho$. The range of various coordinates are as follows:

$$
\begin{aligned}
& -\infty<x^{\mu}<\infty, \quad \text { for } \quad 0 \leq \mu \leq 6, \\
& \pi \leq \rho<\infty, \quad 0 \leq \theta \leq \pi, \quad 0 \leq \phi \leq 2 \pi, \quad 0 \leq \psi \leq 2 \pi .
\end{aligned}
$$

The coordinates $\phi$ and $\psi$ are periodic with period $2 \pi$. Finally, there is an identification of coordinates under a $Z_{2}$ transformation $I$ given by:

$$
I: \quad(\theta, \phi, \psi) \rightarrow(\pi-\theta, \pi+\phi,-\psi),
$$

with all other coordinates remaining fixed.

Near $\rho=\pi$, the functions $f, a, b$ and $c$ behave as

$$
f \simeq-1, \quad a \simeq 2(\rho-\pi), \quad b \simeq \pi, \quad c \simeq-\pi
$$


Since $a$ vanishes at $\rho=\pi$, the metric appears to be singular there. However if we introduce new angular coordinates $\widetilde{\theta}, \widetilde{\phi}$ and $\widetilde{\psi}$ through the relations

$$
\begin{aligned}
\sigma_{1} & =d \widetilde{\psi}+\cos \widetilde{\theta} d \widetilde{\phi}, \\
\sigma_{2} & =-\sin \widetilde{\psi} d \widetilde{\theta}+\cos \widetilde{\psi} \sin \widetilde{\theta} d \widetilde{\phi}, \\
\sigma_{3} & =\cos \widetilde{\psi} d \widetilde{\theta}+\sin \widetilde{\psi} \sin \widetilde{\theta} d \widetilde{\phi},
\end{aligned}
$$

and define $\widetilde{\rho}=\rho-\pi$, then near $\rho=\pi$ the Atiyah-Hitchin metric in this coordinate system takes the form:

$$
d s_{A H}^{2} \simeq d \widetilde{\rho}^{2}+4 \widetilde{\rho}^{2}(d \widetilde{\psi}+\cos \widetilde{\theta} d \widetilde{\phi})^{2}+\pi^{2}\left(d \widetilde{\theta}^{2}+\sin ^{2} \widetilde{\theta} d \widetilde{\phi}^{2}\right) .
$$

Furthermore the transformation $I$ acts on the new coordinates as

$$
I: \quad(\widetilde{\theta}, \widetilde{\phi}, \widetilde{\psi}) \rightarrow(\widetilde{\theta}, \widetilde{\phi}, \widetilde{\psi}+\pi) .
$$

Thus identification under $I$ makes $\tilde{\psi}$ an angular variable with period $\pi$. From (2.9) we now see that for fixed $(\widetilde{\theta}, \widetilde{\phi}),(\widetilde{\rho}, 2 \widetilde{\psi})$ describes a plane near the origin in polar coordinates, and for fixed $(\widetilde{\rho}, \widetilde{\psi}),(\widetilde{\theta}, \widetilde{\phi})$ describes a sphere of radius $\pi$. Thus near $\widetilde{\rho}=0$ the space is non-singular, and locally has the structure or $R^{2} \times S^{2}$. The sphere $S^{2}$ parametrized by $(\widetilde{\theta}, \widetilde{\phi})$ is a non-trivial two cycle (of minimal area) in this space, and is known as the 'Bolt'. We shall denote this by $C_{0}$. In the metric (2.2) this sphere has radius $\pi R / 2$ and hence has area:

$$
A=\pi^{3} R^{2},
$$

measured in the eleven dimensional metric.

The space $\overline{\mathcal{N}}$ spanned by $\rho, \theta, \phi$ and $\psi$ has a self-dual harmonic two form $\omega_{0}$ given by 16, 17, 18

$$
\omega_{0}=K_{0} \exp \left(-\int_{\pi}^{\rho} d \rho^{\prime} \frac{f\left(\rho^{\prime}\right) a\left(\rho^{\prime}\right)}{b\left(\rho^{\prime}\right) c\left(\rho^{\prime}\right)}\right)\left(d \sigma_{1}-\frac{f(\rho) a(\rho)}{b(\rho) c(\rho)} d \rho \wedge \sigma_{1}\right),
$$

where $K_{0}$ is a normalization constant. It is natural to choose $K_{0}$ such that $\int_{C_{0}} \omega_{0}=1$.

Given this description of the O6-plane - D6-brane system in M-theory, we now try to address the question we had posed. First we need to understand the origin of the $\mathrm{SO}(2)$ gauge field in this system. This is found as follows. If $C$ denotes the three form gauge field of M-theory, then we can decompose this as

$$
C=\omega_{0}(y) \wedge A(x)+\ldots
$$


where $y$ denotes the coordinate on the Atiyah-Hitchin space, $x$ denotes the coordinates on $R^{6,1}, A$ is a one form field on $R^{6,1}$ and ... denotes an infinite number of other terms which are not relevant for the present analysis. $A(x)$ is the world-volume $\mathrm{SO}(2)$ gauge field that we have been looking for.

The next question will be: how do we get states charged under $A_{\mu}$ ? Since a membrane is charged under the three form field $C$, a membrane wrapped around the cycle $C_{0}$ will carry an $A_{\mu}$ charge proportional to $\int_{C_{0}} \omega_{0}$. Thus this is the M-theory description of the open string state that carries charge under the $\mathrm{SO}(2)$ gauge field. Although our analysis does not show directly that it carries the right amount of $\mathrm{SO}(2)$ charge, it is clear that this is the state carrying minimal $\mathrm{SO}(2)$ charge in the M-theory description, and hence must have the right charge. The mass of this state is given by the product of the membrane tension $T_{M}$ and the area of the two cycle $C_{0}$ :

$$
m \simeq T_{M} A=T_{M} \pi^{3} R^{2}
$$

Note that this mass formula will get modified by quantum fluctuations of the membrane. However, as long as $R$ is large, the classical contribution (2.14) to the mass will be the dominant contribution, and (2.14) will be reliable. There will also be corrections to the mass formula due to membrane self-interaction since we have a curved membrane instead of a planar membrane. However, as long as $R$ is large, the contribution due to such interactions will also be small.

Let us briefly set $\alpha^{\prime}=1$ and work in the normalization convention of [19. In this convention, the membrane tension measured in the eleven dimensional metric is given by

$$
T_{M}=\frac{1}{(2 \pi)^{2}}
$$

On the other hand, $R$ measured in the eleven dimensional metric is related to the type IIA coupling constant through the relation[3]:

$$
R=g_{S}^{2 / 3}
$$

Finally the mass measured in the type IIA string metric is obtained from that measured in the eleven dimensional metric by multiplying the latter by a factor of $R^{-1 / 2}=g_{S}^{-1 / 3}[3]$.

\footnotetext{
${ }^{4}$ This can be seen by noting that upon compactification on a circle of radius $R$, the string tension measured in the eleven dimensional metric is given by $2 \pi R T_{M}$. Using the relation between the eleven dimensional metric and the ten dimensional string metric we get the string tension measured in ten dimensional string metric to be $2 \pi T_{M}$, which must be equal to $(2 \pi)^{-1}$ for $\alpha^{\prime}=1$.
} 
Thus the mass of the state, measured in the type IIA string metric, is given by $\left(\pi g_{S} / 4\right)$. Finally, restoring the factor of $\alpha^{\prime}$ by dimensional analysis, we arrive at the final answer for the mass of the charged open string state in the strong coupling limit of type IIA string theory:

$$
m \simeq \frac{\pi}{4}\left(\alpha^{\prime}\right)^{-1 / 2} g_{S}
$$

Thus for $p=6, f_{p}\left(g_{S}\right)$ defined in eq.(1.2) is given by $\left(\pi g_{S} / 4\right)$ for large $g_{S}$.

\section{Dirichlet 7-brane on Top of an Orientifold 7-plane}

In this case the configuration under study is a single D7-brane on top of a single O7-plane in type IIB string theory. We shall use the normalization convention of ref. 119 and set $\alpha^{\prime}=1$. Let us denote by $\lambda$ the complex scalar field

$$
\lambda=a+i e^{-\phi} \equiv \lambda_{1}+i \lambda_{2}
$$

where $a$ is the RR sector scalar field and $\phi$ is the dilaton. The string coupling constant $g_{S}$ is related to the imaginary part of $\lambda$ via the relation:

$$
g_{S}=\left(\lambda_{2}\right)^{-1} \text {. }
$$

The O7 plane in type IIB string theory can be described as the quotient space:

$$
R^{7,1} \times\left(R^{2} /(-1)^{F_{L}} \cdot \Omega \cdot \mathcal{I}_{2}\right) .
$$

Since the space transverse to the O7-plane is two dimensional, we can label this space by a single complex coordinate $z$. Let us also denote by $w$ the coordinate on the covering space $R^{2}$. Thus $w$ is related to $z$ by the relation $z=w^{2}$.

We shall be interested in studying a configuration of an O7-plane and a D7-brane situated at $z=0$. In the weak coupling limit the background around the D7-brane O7-plane system is given by

$$
\begin{gathered}
\lambda=-\frac{3}{2 \pi i} \ln \left(z / L^{2}\right)=-\frac{3}{\pi i} \ln (w / L) \\
d s_{c a n}^{2} \equiv \sum_{M, N=0}^{9} g_{M N}^{(c a n)} d x^{M} d x^{N}=\sum_{\mu, \nu=0}^{7} \eta_{\mu \nu} d x^{\mu} d x^{\nu}+\lambda_{2} d w d \bar{w} \\
=\sum_{\mu, \nu=0}^{7} \eta_{\mu \nu} d x^{\mu} d x^{\nu}+\frac{1}{4} \lambda_{2}|z|^{-1} d z d \bar{z}
\end{gathered}
$$


where $L$ is a constant, and $g_{M N}^{(\text {can })}$ denotes the ten dimensional canonical metric of the type IIB string theory. The factor of -3 in the expression for $\lambda$ reflects the fact that the O7-D7 system carries a total of -3 units of RR charge, of which 1 unit comes from the D7-brane and -4 units come from the O7-plane. Note that since $\lambda$ does not go to a constant asymptotically, one cannot use the asymptotic string coupling constant as a parameter. Instead we can use $L$ as the parameter measuring the strength of the string coupling constant. As seen from (3.4), at a large but fixed value of $z, \lambda_{2}$ decreases as $L$ increases. Thus large $L$ will correspond to strong coupling limit. Alternatively, one can 'regularize' the asymptotic behaviour of $\lambda$ by placing three D7-branes at $z=z_{0}\left(\equiv w_{0}^{2}\right)$ for some very large $\left|z_{0}\right|$. Now the background $\lambda$ in the weak coupling region is given by,

$$
\lambda=-\frac{3}{2 \pi i} \ln \frac{w^{2}}{w^{2}-w_{0}^{2}}+i g_{S}^{-1}
$$

where $g_{S}$ is the asymptotic coupling constant. Thus for $|w|<<\left|w_{0}\right|$, but sufficiently far away from the origin so that $\lambda_{2}$ is large, we get

$$
\lambda \simeq-\frac{3}{2 \pi i} \ln \frac{w^{2}}{\left(-w_{0}^{2}\right)}+i g_{S}^{-1} .
$$

(3.7) has the same form as (3.4) if we choose

$$
L=i w_{0} \exp \left(-\frac{\pi}{3 g_{S}}\right)
$$

We can now take $g_{S}$ as our independent parameter instead of $L$. However, since (3.6), (3.7) are valid only when $\lambda_{2}$ is large, we need to keep $g_{S}$ small. The theory is made strongly coupled by taking $\left|w_{0}\right|$ large.

Since the O7-plane and the D7-brane are coincident, the classical mass of an open string starting on the D7-brane, going around the O7-plane and ending on the D7-brane vanishes. However, once non-perturbative effects are taken into account, the coincident O7-plane - D7-brane system is replaced by a system of three mutually non-local seven branes 20, 21, 22], the location and type of these seven branes being given by the SeibergWitten solution for $\mathrm{N}=2$ supersymmetric $\mathrm{SU}(2)$ gauge theory with one massless hypermultiplet in the fundamental representation. The open string state under study is represented by an appropriate network 23 of open strings stretched between these seven branes 24, 25, 26, 27, 28, 29, 30, 31. The mass of this network can be found by adding up the masses of each segment of this network. In the $w$ coordinate system the seven branes 
are located in the region where $\lambda_{2} \sim 1$, i.e. at $|w| \sim|L|$. Since in this region $\lambda$ is of order unity, $g_{w \bar{w}}^{(c a n)}$ as well as the tension of any string of type $(p, q)$ is also of order unity. Thus the open string network has size and mass of order $|L|$ measured in the canonical metric. This gives a mass formula of the form:

$$
m \simeq C|L|
$$

where $C$ is a numerical constant. This mass formula gets corrected by quantum fluctuations on the stretched string network, and also due to the interaction between different segments of the network. However, as long as $|L|$ is large, the classical contribution to the mass of the stretched string network dominates over these corrections, and hence (3.9) remains valid.

Computation of the precise value of $C$ requires identifying the open string network that represents the lightest state carrying $\mathrm{SO}(2)$ charge. This has been carried out in the appendix. The answer is

$$
C=\frac{\Gamma\left(\frac{1}{3}\right)}{4 \pi\left(\Gamma\left(\frac{7}{6}\right)\right)^{2}} 2^{2 / 3} 3^{1 / 2} .
$$

Using eq.(3.8) we can express (3.9) as

$$
m \simeq C\left|w_{0}\right| \exp \left(-\frac{\pi}{3 g_{S}}\right) .
$$

In order to calculate $f_{p}\left(g_{S}\right)$ defined in (1.2) for $p=7$, we need to express this in string metric. In ten dimensional type IIB string theory the string metric is obtained from the

canonical metric by multiplying the latter by a factor of $g_{S}^{1 / 2}$. This gives the following expression for the mass of the charged open string state on the D7-O7 world-volume:

$$
m \simeq C\left(\alpha^{\prime}\right)^{-1 / 2}\left|\frac{w_{0}}{\sqrt{\alpha^{\prime}}}\right|\left(g_{S}\right)^{-1 / 4} \exp \left(-\frac{\pi}{3 g_{S}}\right) .
$$

Note that we have restored the factors of $\alpha^{\prime}$. Comparison with (1.2) gives:

$$
f_{7} \simeq C\left|\frac{w_{0}}{\sqrt{\alpha^{\prime}}}\right|\left(g_{S}\right)^{-1 / 4} \exp \left(-\frac{\pi}{3 g_{S}}\right) .
$$

\section{Dirichlet 4-brane on Top of an Orientifold 4-plane}

In this case the theory is type IIA string theory, and the orientifold 4-plane is the space:

$$
R^{4,1} \times\left(R^{5} / \Omega \cdot \mathcal{I}_{5}\right)
$$


The strong coupling limit of this theory is M-theory compactified on a circle $S^{1}$ of large radius $R$. In this limit the Dirichlet 4-brane is described by a five-brane of M-theory with one direction tangential to the circle. The orientifold 4-plane, on the other hand, is described by the space:

$$
R^{4,1} \times S^{1} \times\left(R^{5} / \sigma \cdot \mathcal{I}_{5}\right),
$$

where $\sigma$ denotes the transformation that changes the sign of the anti-symmetric tensor field of M-theory. Various properties of this orbifold were analyzed in refs. 32, 33].

On the world-volume of the five brane there is a massless rank two anti-symmetric tensor field $\mathcal{B}_{\mu \nu}$. Upon compactification on $S^{1}$, this gives the gauge field living on the 4-brane world-volume. Thus the states charged under this gauge field can be constructed by taking a string on the five-brane world-volume carrying $\mathcal{B}_{\mu \nu}$ charge, and wrapping it on $S^{1}$. This string in turn may be constructed as follows. First consider the case where the five-brane is away from the orbifold fixed plane but parallel to it. Since membranes can end on five branes [34, 35], we can consider a membrane configuration that starts on the five brane, goes around the orbifold plane, and ends on the five-brane. This describes a string like state on the five-brane world-volume carrying $\mathcal{B}_{\mu \nu}$ charge, with classical contribution to the tension proportional to the distance between the five-brane and the orbifold plane. If we now consider the limit when the distance between the five-brane and the orbifold plane goes to zero, the classical contribution to the tension goes to zero, but there is a quantum contribution which, on purely dimensional grounds, must be of order $m_{p}^{2}$, where $m_{p}$ is the eleven dimensional Planck mass. This will describe a non-BPS string on the world-volume of the 5-brane - orbifold plane system.

As in section 2, let us briefly set $\alpha^{\prime}=1$. As argued in that section, this would correspond to setting the membrane tension $T_{M}$, measured in the eleven dimensional metric, to $1 / 4 \pi^{2}$. Since $T_{M} \propto m_{p}^{3}$, this would also correspond to setting $m_{p}$, measured in the eleven dimensional metric, to a number of order unity. Thus in this metric the tension of the non-BPS string carrying $\mathcal{B}_{\mu \nu}$ charge is of order unity, and the mass of the state obtained by wrapping this string around a circle of radius $R$ is of order $R$. Using eq. (2.16) we see that this gives a mass of order $g_{S}^{2 / 3}$. In order to convert it to the mass measured in string metric, we need to multiply it by a factor of $g_{S}^{-1 / 3}$. This gives the follwing expression for the mass of the lightest state charged under the $\mathrm{SO}(2)$ gauge field

\footnotetext{
${ }^{5}$ In the covering space this corresponds to a membrane with one end terminating on the five-brane and the other end terminating on the image of the five brane under the $Z_{2}$ transformation $\sigma \cdot \mathcal{I}_{5}$.
} 
on the D4-O4 brane world volume:

$$
m \simeq K\left(\alpha^{\prime}\right)^{-1 / 2} g_{S}^{1 / 3}
$$

where $K$ is a numerical constant. Note that we have restored the factor of $\alpha^{\prime}$ in (4.3). Determining the value of $K$ will require computing the tension of the non-BPS string through a detailed analysis of the dynamics of the membrane stretched between the fivebrane and the orbifold plane. This is beyond the scope of the present analysis.

Comparing (4.3) with (1.2) we arrive at the following result for the strong coupling behaviour of $f_{4}\left(g_{S}\right)$ :

$$
f_{4}\left(g_{S}\right) \simeq K g_{S}^{1 / 3}
$$

\section{$5 \quad$ Other $\mathbf{D} p$-brane - $\mathrm{O} p$-plane Systems}

For $p=5$, the theory under consideration is type IIB string theory, and the orientifold 5 -plane is given by the quotient:

$$
R^{5,1} \times\left(R^{4} / \mathcal{I}_{4} \cdot \Omega\right)
$$

In studying the strong coupling limit of the coincident O5-D5 system, we can use the S-duality transformation of type IIB string theory. This converts the transformation $\Omega$ to $(-1)^{F_{L}}[37$. Thus the strong coupling dual of the O5-plane is

$$
R^{5,1} \times\left(R^{4} / \mathcal{I}_{4} \cdot(-1)^{F_{L}}\right)
$$

It turns out that this orbifold actually represents the dual of an O5-plane - D5-brane system 36, 37] and not of an isolated O5-plane. To see this note that the twisted sector states of this orbifold give rise to a $U(1)$ gauge field living on the fixed plane. Since an isolated orientifold plane does not have any massless field living on its world-volume, (5.1) must be dual to an O5-plane - D5-brane system. Indeed, the full spectrum of massless twisted sector states in the orbifold (5.2) consists of a matter multiplet of the $(1,1)$ supersymmetry algebra in six dimensions, exactly matching the spectrum of massless states on the world-volume of the O5-D5 system.

Once we have identified the strong coupling dual of the O5-brane - D5-plane configuration, we can formulate the problem under study in a precise manner. We need to find the lightest state in this orbifold theory which is charged under the gauge field coming 
from the twisted sector of the orbifold. Unfortunately, even though the question is well posed, the answer is not known. We shall speculate on a possible answer in the next section.

For $p=3$, the theory under consideration is again type IIB string theory, and the orientifold 3-plane is given by the quotient:

$$
R^{3,1} \times\left(R^{6} / \mathcal{I}_{6} \cdot \Omega \cdot(-1)^{F_{L}}\right)
$$

In studying the strong coupling limit of the coincident O3-D3 system, we can use the Sduality transformation of type IIB string theory. This leaves invariant $\Omega \cdot(-1)^{F_{L}}$, and also the D3-brane. Thus the D3-O3 system is self-dual. However, this duality transformation induces an electric-magnetic duality transformation on the world-volume theory 38, 35], so that the state electrically charged under $\mathrm{SO}(2)$ gets mapped to a state magnetically charged under $\mathrm{SO}(2)$. Thus the problem of determining the mass of the lightest electrically charged state in the strong coupling limit gets mapped to the problem of finding the mass of the lightest magnetically charged state on the world-volume of the D3-O3 system in the weak couping limit. Again this problem, although well-posed, has not been solved.

For $p<3$ a state carrying $\mathrm{SO}(2)$ gauge charge has infinite energy due to the long range gauge field strength that it produces on the brane. Thus the mass of such a state is not well defined. This leaves only the case of $p=8$. In this case the theory under study is type IIA string theory, and its strong coupling limit is described by M-theory; but unfortunately neither the D8-brane nor the O8-plane has been understood well from the point of view of M-theory. Thus we cannot use the M-theory description of the theory to analyze the spectrum of stable non-supersymmetric states in the world-volume of this theory.

\section{Some Speculations about the Missing States}

In this section we shall make some speculations about the origin of the missing states. First let us recollect that in the examples discussed in this paper, there are three cases where the required charged states were not found. They are as follows:

1. In the weakly coupled type I string theory, we should have solitonic states transforming in the spinor representation of the gauge group. These states have not been constructed explicitly. 
2. On the orbifold plane $R^{5,1} \times\left(R^{4} /(-1)^{F_{L}} \cdot \mathcal{I}_{4}\right)$ in type IIB string theory, we should have states carrying charge under the gauge field originating in the twisted sector of the orbifold. These states have not been found.

3. Finally, in the system containing a D3-brane on top of an O3-plane, we expect to find states which are magnetically charged under the $\mathrm{SO}(2)$ gauge field living on the world-volume of the system. These states have also not been found explicitly.

There are two features common between these three cases. First of all, in each case the theory under consideration is an orbifold / orientifold of type IIB string theory. (We are regarding type I theory as type IIB theory modded out by the transformation $\Omega$.) Second, in each case, following the duality transformation that led to the existence of these states, we see that the states under consideration should correspond to microscopic closed / open D-strings. In the first case, the states in the spinor representation in the original $\operatorname{Spin}(32) / Z_{2}$ heterotic string theory are elementary string states and hence can be regarded as coming from small loops of fundamental strings. Thus in the dual type I theory these should be represented as small loops of D-strings. In the second and the third case, the states under consideration in the original type IIB theory come from microscopic open strings with ends lying on the D-brane. Since the strong-weak coupling duality converts a fundamental string to a D-string, we would expect that in the dual type IIB theory these states should be represented as microscopic open D-strings.

Although we do not know how to analyze microscopic D-strings, there is one qualitative feature of these states that we can guess - these states must be able to support configurations where one end of a fundamental string is stuck to the state. In other words, these states must have properties similar to a D0-brane! Of course in type IIB theory itself D0-branes cannot be defined consistently; however one might conjecture that in the presence of orientifold / orbifold planes we can define analogs of D0-branes in type IIB theory if the D0-branes are embedded in the orientifold / orbifold plane.

There are also other arguments one can give in support of this conjecture. For example a D0-brane of this kind in type I theory will support open strings with one end stuck to the 0-brane, and other end free with Neumann boundary condition. These will transform in the fundamental representation of $\mathrm{SO}(32)$ due to the Chan-Paton factors associated with the free end, and quantizing the zero energy fermionic states of this string will indeed give states of the D0-brane in the spinor representation of the gauge group. (Of course the 
origin of the GSO projection in this case is somewhat obscure, and presumably related to the existence of a subtle $Z_{2}$ gauge symmetry on the D0-brane world-volume[6].) In the second example, a single D0-brane situated on the orbifold 5-plane will most likely carry charge under the gauge field coming from the twisted sector as in the case of [39]. This will again be in accordance with the expected property of these 0-branes.

Conformal field theories describing excitations on these D0-branes are certainly going to be more complicated than those for supersymmetric D-branes. Construction of these conformal field theories remains a challenging problem for the future.

Acknowledgement: A preliminary version of this work was reported in the string workshop at the Institute of Physics, Bhubaneswar. I wish to thank the workshop participants for their comments.

\section{A Computation of the Coefficient $C$}

In this appendix we shall compute the coefficient $C$ introduced in section 3 . We begin by introducing some notations. A $(p, q)$ string will denote a bound state of $p$ fundamental strings and $q$ D-strings, and a $(p, q)$ seven brane will denote a seven brane on which a $(p, q)$ string can end. Thus in this notation a D7-brane will be labelled as a $(1,0)$ seven brane. The $\mathrm{SL}(2, \mathrm{Z})$ monodromy around a $(p, q)$ seven brane is given by

$$
M_{p, q}=\left(\begin{array}{cc}
1-p q & p^{2} \\
-q^{2} & 1+p q
\end{array}\right)
$$

Our convention is the same as that in ref. [27]; as an $(r, s)$ string / 7-brane crosses the cut associated with a $(p, q)$ seven brane in the anti-clockwise direction, it gets converted to an $M_{p, q}\left(\begin{array}{l}r \\ s\end{array}\right)$ string / 7-brane.

Let us now turn to the O7-D7 system under study. First consider the case where the D7-brane is far away from the O7-plane. In this case the D7-brane is represented as a $(1,0)$ 7-brane, whereas the O7-plane splits into a pair of parallel $(0,1)$ and $(2,1) 7$-branes 20] as shown in Fig.1. In order to make this description meaningful, we must describe the path along which the asymptotic observer views different 7-branes. This has been indicated by the dashed lines in Fig.1. The same configuration can be viewed as a collection of a $(1,1)$, $(0,1)$ and $(2,1) 7$-branes by changing the path along which we view the branes. This has been shown in Fig.2. 


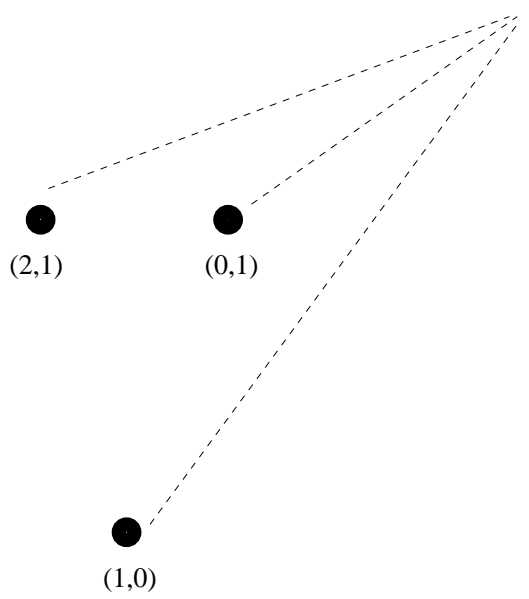

Figure 1: D7-O7 system with D7-brane far away from the O7-plane. The dashed lines indicate the path along which the seven branes are viewed.

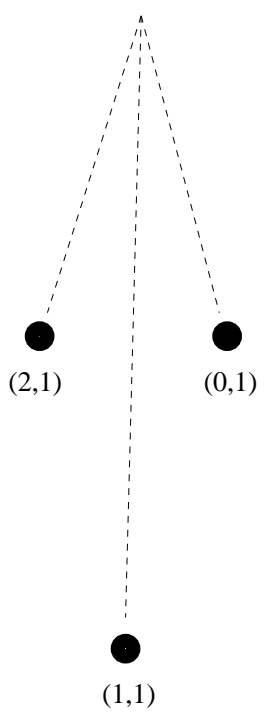

Figure 2: A different view of the configuration shown in Fig.11.

A state on the D7-O7 world volume theory, carrying charge under the world-volume gauge field, is represented by an open string starting on the (1,1) 7-brane, going around the $(2,1)$ and the $(0,1) 7$-branes, and coming back to the $(1,1)$ brane, as shown in Fig. 3 . 


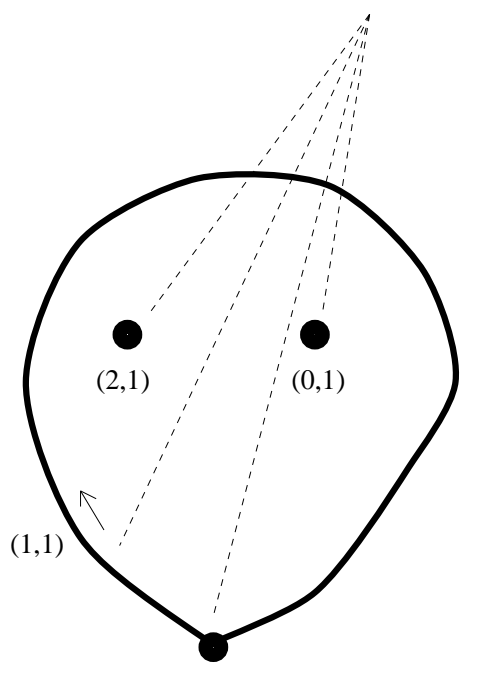

$(1,1)$

Figure 3: An open string configuration representing a state carrying $\mathrm{SO}(2)$ charge.

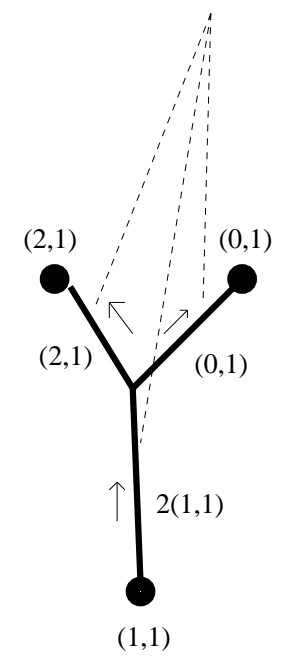

Figure 4: The minimal energy configuration of open string network representing a state carrying $\mathrm{SO}(2)$ charge. Although in this figure we have displayed the open strings to be lying along straight lines, they actually lie along geodesics described in eq. A.9). 
However, as was pointed out in ref.[27], this configuration does not represent a geodesic, and hence does not minimize the energy stored in the string. The minimal energy configuration 29] is shown in Fig. Th. This configuration can be obtained from the one in Fig. 3 by deforming the open string loop through the $(2,1)$ and the $(0,1)$ seven branes. According to the rules of ref. [27, during this process new open string prongs connecting the original string and the 7-branes are generated, as has been shown in Fig.4. Note that the string junction where the $(2,1),(1,1)$ and the two $(1,1)$ strings meet conserve charge, as required. However, in checking charge conservation one must ensure that all the strings meeting at a junction are viewed along the same path, or along paths which are continuously deformable to one another without passing through a seven-brane. This is satisfied in Fig. 4 , as the three dashed lines along which the three types of strings are viewed, are continuously deformable to one another. The precise location of the string junction, as well as the path followed by the different segments of the network is determined by minimizing the energy of this configuration following refs. [24, 29]. If we naively follow the arguments of ref. 29] by replacing their three brane by a D7-brane, we would conclude that this configuration is supersymmetric. However, a more careful analysis [31] shows that it does not represent a BPS state. This is in accordance with the expected property of this state.

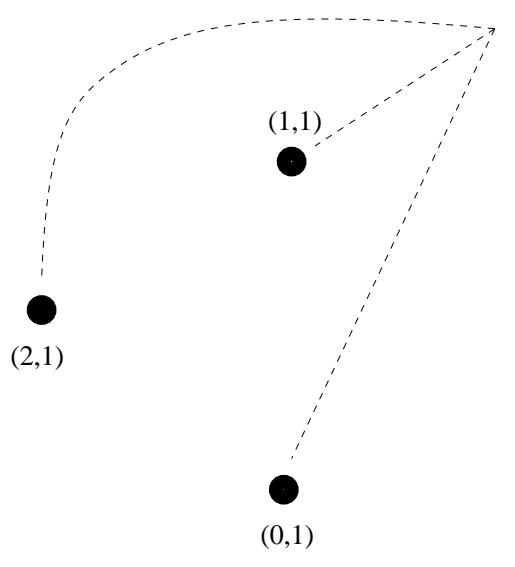

Figure 5: The 7-brane configuration for coincident D7-O7 system. 


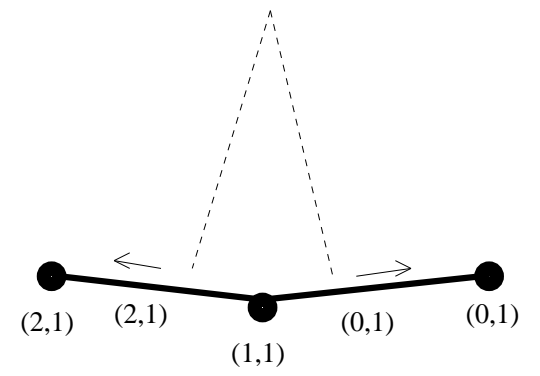

Figure 6: The open string network representing the lightest charged state at critical distance.

Now consider reducing the distance between the D7-brane and the O7-plane to zero. In this limit the configuration of 7-branes is given by the Seiberg-Witten result for $\mathrm{N}=2$ supersymmetric $\mathrm{SU}(2)$ gauge theory with one flavour of massless quark hypermultiplet in the fundamental representation [40]. This configuration is displayed in Fig.5 and is reached from Fig.2 by a continuous deformation moving the $(1,1)$ seven brane through the other two seven branes. The question that we would like to address is: what configuration does the string network shown in Fig. 14 evolve to during this process? For this we try to follow this configuration as the distance between the O7-plane and D7-brane decreases continuously. At some point during this process, which we might call the critical distance, the length of the $(1,1)$ portion of the network goes to zero, as shown in Fig.6. The question is what happens to this network when we reduce the distance even further?

There are two possibilities we can consider. The first possibility, which is a conservative picture, is that as the distance parameter is reduced below the critical distance, the $(2,1)$ and the $(0,1)$ strings are dragged along with the $(1,1)$ seven brane as shown in Fig. 7 . According to this picture, the lightest charged state on the D7-O7 world volume for coincident D7-O7 system is given by the open string network shown in Fig.8. This is certainly an allowed configuration, and hence the mass of this state gives an upper bound to the mass of the lightest charged state in the world-volume theory.

A more radical possibility for what happens at less than critical distance has been shown in Fig.9. First of all, note that in this figure the original $(2,1)$ seven brane and

\footnotetext{
${ }^{6}$ Since the O7-plane splits into two branes, one cannot make this distance vanish. What we mean by this limit is that the parameter that labels the classical distance between the plane and the brane goes to zero.
} 


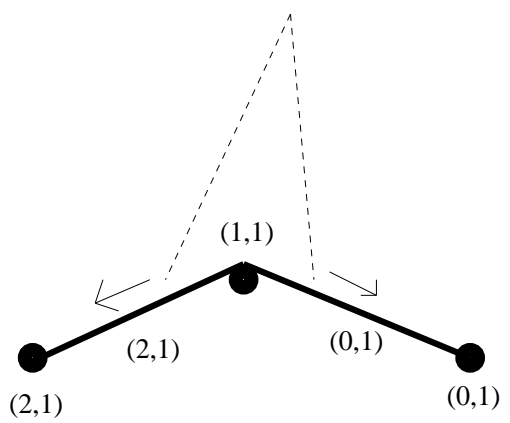

Figure 7: A possible open string network representing lightest charged state at less than the critical distance.

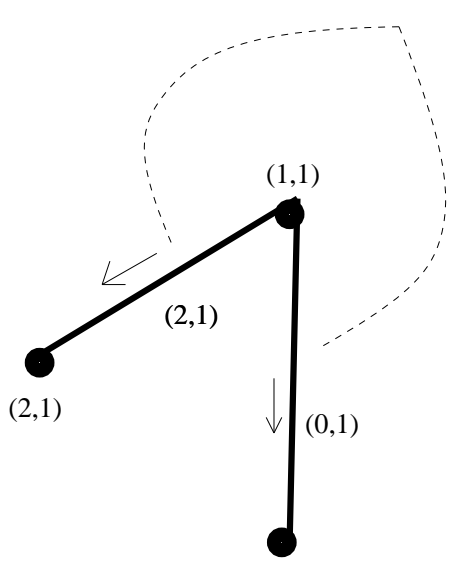

$(0,1)$

Figure 8: A possible open string network representing lightest charged state for coincident D7-O7 system.

the attached $(2,1)$ string are viewed along a different path in order to be able to check charge conservation at the string junction. Thus they appear as $(1,0)$ seven brane and $(1,0)$ string respectively. The second point to note is that charge conservation at the string junction demands that now there is a single $(1,1)$ string coming out the $(1,1)$ seven brane instead of two $(1,1)$ strings as in Fig. 4 . One way to interprete this phenomenon is that the original $(2,1)$ string, in the process of passing through the $(1,1)$ seven brane, has developed a $(1,1)$ prong connecting it to the $(1,1)$ seven brane. This new prong cancels 


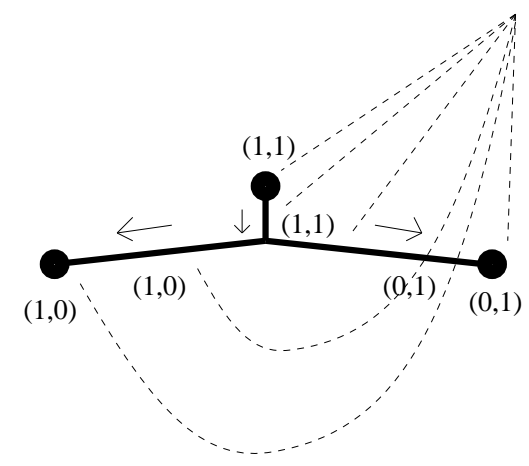

Figure 9: Another possible open string network representing lightest charged state at less than critical distance.

the charge of one of the original $(1,1)$ strings, leaving behind only one $(1,1)$ string joining the $(1,1)$ seven brane to the triple string junction. Deformations of this kind, leading from Fig.4 to Fig.9, have been discussed recently in [31.

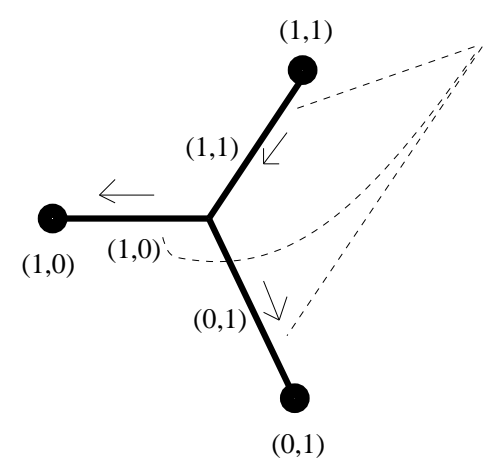

Figure 10: The open string network representing lightest charged state for coincident D7-O7 system.

Naively the configuration displayed in Fig.9 seem to carry only half the $\mathrm{SO}(2)$ charge as the configuration of Fig. 4 , since only one $(1,1)$ string comes out of the $(1,1)$ seven brane. However, since the configuration of Fig.9 is obtained by continuous deformation from the configuration of Fig.4, we would expect Fig.9 to represent a state carrying the same $\mathrm{SO}(2)$ charge as Fig.4. Also by embedding the D7-O7 configuration under study 
into a complete orientifold of type IIB on $R^{7,1} \times T^{2} /(-1)^{F_{L}} \cdot \Omega \cdot \mathcal{I}_{2}$, it is easy to see that a state carrying half integral $\mathrm{SO}(2)$ charge (and no other charge) cannot exist for purely group theoretic reason. Thus the most likely interpretation of the state displayed in Fig.9 is that it represents a state carrying unit $\mathrm{SO}(2)$ charge. This charge assignment cannot be directly read out from examining how many $(1,1)$ open strings end on the $(1,1)$ seven brane. Similar situation was encountered in ref. [27] in searching for $E_{n}$ gauge boson in the orientifold theory. According to this view, the lightest open string state carrying unit $\mathrm{SO}(2)$ charge for coincident D7-O7 system is given by the configuration shown in Fig.10. The $Z_{3}$ symmetry of the brane configuration 40 implies that the three string vertex must be located at the center of the equilateral triangle whose vertices are the locations of the three seven branes. The coefficient $C$ is determined by calculating the mass of the open string network of Fig.10. This is the problem to which we shall now turn.

The non-perturbative effects modify the background fields (3.4) (3.5) to 20

$$
\begin{gathered}
\lambda(z)=\left(\frac{d a_{D}}{d z}\right) /\left(\frac{d a}{d z}\right), \\
d s_{c a n}^{2}=2 \lambda_{2} d a d \bar{a}+\sum_{\mu, \nu=0}^{7} \eta_{\mu \nu} d x^{\mu} d x^{\nu},
\end{gathered}
$$

where $a$ and $a_{D}$ are integrals of Seiberg-Witten differential 40, appropriately normalized so that the background described in (A.2), (A.3) matches the one given in (3.4), (3.5) for large $|z|$. This can be achieved by choosing

$$
a(z)=\widetilde{L} a^{(1)}\left(z / \widetilde{L}^{2}\right), \quad a_{D}(z)=\widetilde{L} a_{D}^{(1)}\left(z / \widetilde{L}^{2}\right)
$$

where $a^{(1)}$ and $a_{D}^{(1)}$ are the functions defined in ref. 41] and $\widetilde{L}$ is a constant to be determined shortly. $a^{(1)}(u)$ and $a_{D}^{(1)}(u)$ have the following asymptotic behaviour for large $|u|$ :

$$
\begin{aligned}
a^{(1)}(u) & \simeq \sqrt{\frac{u}{2}} \\
a_{D}^{(1)}(u) & \simeq \frac{3 i}{2 \pi} \sqrt{\frac{u}{2}}\left[\ln u+\frac{4}{3} \ln 2-2+\ln 3+\frac{i \pi}{3}\right] .
\end{aligned}
$$

Using eqs.(A.4), (A.5) we see that the background described in (A.2), (A.3) matches the one given in (3.4), (3.5) for large $|z|$ if we choose:

$$
\widetilde{L}=2^{2 / 3} 3^{1 / 2} e^{i \pi / 6} L
$$


In units where $\alpha^{\prime}$ is set equal to unity, the tension of a $(p, q)$ string, measured in the canonical metric, is given by 42

$$
T(p, q)=\frac{1}{2 \pi} \frac{1}{\sqrt{\lambda_{2}}}|p+q \lambda| .
$$

Thus the mass of a $(p, q)$ string stretched between $z$ and $z+d z$ is given by

$$
T(p, q) \sqrt{g_{z \bar{z}}}|d z|=\frac{1}{\sqrt{2} \pi}\left|p d a+q d a_{D}\right| .
$$

As a result, for a $(p, q)$ string stretched between two fixed points in the $z$ plane, the minimum energy configuration will require the string to lie along the curve[24]

$$
\left(p a+q a_{D}\right)=C_{1}+C_{2} t
$$

where $C_{1}$ and $C_{2}$ are constants and $t$ is a real parameter labelling points on the curve. We shall call this the $(p, q)$ geodesic. This gives the following expression for the mass of a $(p, q)$ string stretched between two points $z_{1}$ and $z_{2}$ 24]:

$\frac{1}{\sqrt{2} \pi}\left|p a\left(z_{1}\right)+q a_{D}\left(z_{1}\right)-p a\left(z_{2}\right)-q a_{D}\left(z_{2}\right)\right|=\frac{|\widetilde{L}|}{\sqrt{2} \pi}\left|p a^{(1)}\left(u_{1}\right)+q a_{D}^{(1)}\left(u_{1}\right)-p a^{(1)}\left(u_{2}\right)-q a_{D}^{(1)}\left(u_{2}\right)\right|$,

where $u=z / \widetilde{L}^{2}$. The mass of the open string configuration in Fig.10 can be calculated simply by adding up the contributions from the different segments of the string network. The calculation is further simplified by noting that the combination $\left(p a+q a_{D}\right)$ vanishes at the location of the $(p, q)$ seven brane.

From 41] we learn that the $(1,0),(0,1)$ and $(1,1)$ seven branes in Fig.10 are located at $u=-1, u=e^{-i \pi / 3}$ and $u=e^{i \pi / 3}$ respectively. Thus due to the $Z_{3}$ symmetry of this configuration, the triple string junction in this figure is located at $u=0$. Using the same symmetry we can also conclude that the three open string segments in this diagram have equal mass. Thus the mass of the open string configuration displayed in Fig.10 is given by:

$$
m=\frac{1}{\sqrt{2} \pi}|\widetilde{L}|\left|3 a_{D}^{(1)}(0)\right|
$$

According to [41] $\left|a_{D}^{(1)}(0)\right|$ is given by $\sqrt{2} F\left(\frac{5}{6}, \frac{5}{6} ; 2 ; 1\right) / 12=\sqrt{2} \Gamma\left(\frac{1}{3}\right) / 12\left(\Gamma\left(\frac{7}{6}\right)\right)^{2}$. Using (A.6) we can now rewrite (A.11) as

$$
m=\frac{\Gamma\left(\frac{1}{3}\right)}{4 \pi\left(\Gamma\left(\frac{7}{6}\right)\right)^{2}} \cdot 2^{2 / 3} \cdot 3^{1 / 2} \cdot|L| .
$$


Comparing this with (3.9) we get,

$$
C=\frac{\Gamma\left(\frac{1}{3}\right)}{4 \pi\left(\Gamma\left(\frac{7}{6}\right)\right)^{2}} \cdot 2^{2 / 3} \cdot 3^{1 / 2} .
$$

Since this derivation of the value of $C$ requires the mild assumption that the configuration of Fig. smoothly evolves into the configuration of Fig.10, we shall now also derive an absolute upper bound on the value of $C$ by calculating the mass of the open string configuration of Fig.8. Using the symmetries of this configuration, and eq.(A.10), we see that the mass of this open string configuration is given by:

$$
\frac{1}{\sqrt{2} \pi}|\widetilde{L}|\left|2 a_{D}\left(e^{i \pi / 3}\right)\right|
$$

$\left|2 a_{D}\left(e^{i \pi / 3}\right)\right|$ is equal to $3 \sqrt{2} / \pi[41$. This gives the mass of the open string configuration of Fig. 8 to be

$$
\frac{1}{\pi^{2}} \cdot 2^{2 / 3} \cdot 3^{3 / 2} \cdot|L|
$$

This gives,

$$
C \leq \frac{1}{\pi^{2}} \cdot 2^{2 / 3} \cdot 3^{3 / 2}
$$

\section{References}

[1] E. Witten and D. Olive, Phys. Lett. B78 (1978) 97. .

[2] D. Gross, J. Harvey, E. Martinec and R. Rohm, Phys. Rev. Lett. 54 (1985) 502; Nucl. Phys. B256 (1985) 253; Nucl. Phys. B267 (1986) 75.

[3] E. Witten, Nucl. Phys. B443 (1995) 85 hep-th/9503124.

[4] A. Dabholkar, Phys. Lett. B357 (1995) 307 hep-th/9506160].

[5] C. Hull, Phys. Lett. B357 (1995) 545 hep-th/9506194.

[6] J. Polchinski and E. Witten, Nucl. Phys. B460 (1996) 525 [hep-th/9510169]. .

[7] J. Polchinski, Phys. Rev. Lett. 75 (1995) 4724 hep-th/9510017;

J. Dai, R. Leigh and J. Polchinski, Mod. Phys. Lett. A4 (1989) 2073;

R. Leigh, Mod. Phys. Lett. A4 (1989) 2767;

J. Polchinski, Phys. Rev. D50 (1994) 6041 [hep-th/9407031]. 
[8] A. Sagnotti, 'Open Strings and their Symmetry Groups', Talk at Cargese Summer Inst., 1987;

G. Pradisi and A. Sagnotti, Phys. Lett. B216 (1989) 59;

M. Bianchi, G. Pradisi and A. Sagnotti, Nucl. Phys. B376 (1992) 365;

P. Horava, Nucl. Phys. B327 (1989) 461; Phys. Lett. B231 (1989) 251.

[9] E. Gimon and J. Polchinski, Phys. Rev. D54 (1996) 1667 [hep-th/9601038].

[10] A. Dabholkar, Phys. Lett.B402 (1997) 53 [hep-th/9702050];

A. Dabholkar, G. Mandal and P. Ramadevi, hep-th/9705239.

[11] A. Sen, hep-th/9708002 (published in JHEP electronic journal).

[12] M. Atiyah and N. Hitchin, Phys. Lett. 107A (1985) 21; Phil. Trans. Roy. Soc. Lond. A315 (1985) 459; Geometry and Dynamics of Magnetic Monopoles, Cambridge Univ. Press.

[13] G. Gibbons and N. Manton, Nucl. Phys. B274 (1986) 183.

[14] N. Seiberg and E. Witten, hep-th/9607163;

N. Seiberg, Phys. Lett. B384 (1996) 81 hep-th/9606017.

[15] A. Sen, hep-th/9707123 (published in JHEP electronic journal).

[16] N. Manton and B. Schroers, Annals Phys. 225 (1993) 290.

[17] G. Gibbons and P. Ruback, Comm. Math. Phys. 115 (1988) 267.

[18] A. Sen, Phys. Lett. B329 (1994) 217 hep-th/9402032.

[19] A. Sen, hep-th/9802051.

[20] A. Sen, Nucl. Phys. B475 (1996) 562 [hep-th/9605150].

[21] T. Banks, M. Douglas and N. Seiberg, Phys. Lett. B387 (1996) 278 hep-th/9605199.

[22] K. Dasgupta and S. Mukhi, Phys. Lett. B385 (1996) 125 [hep-th/9606044]. 
[23] O. Aharony, J. Sonnenschein and S. Yankielowicz, Nucl. Phys. B474 (1996) 309 [hep-th/9603009];

J. Schwarz, hep-th/9607201;

O. Aharony and A. Hanany, hep-th/9704170;

O. Aharony, A. Hanany and B. Kol, hep-th/9710116;

K. Dasgupta and S. Mukhi, hep-th/9711094;

A. Sen, hep-th/9711130;

S.-J. Rey and J.-T. Yee, hep-th/9711202;

M. Krogh and S. Lee, hep-th/9712050;

Y. Matsuo and K. Okuyama, hep-th/9712070];

F. Markopoulou and L. Smolin, [hep-th/9712148;

O. Bergman, hep-th/9712211];

B. Kol and J. Rahmfeld, [hep-th/9801067];

S. Bhattacharyya, A. Kumar and S. Mukhopadhyay, hep-th/9801141;

C. Callan and L. Thorlacius, hep-th/9803097;

K. Hashimoto, H. Hata and N. Sasakura, hhep-th/9803127.

[24] A. Sen, Phys. Rev. D55 (1997) 2501 [hep-th/9608005].

[25] A. Johansen, Phys. Lett. B395 (1997) 36 hep-th/9608186.

[26] A. Fayyazuddin, Mod. Phys. Lett. A10 (1995) 2703 [hep-th/9504120].

[27] M. Gaberdiel and B. Zwiebach, [hep-th/9709013];

M. Gaberdiel, T. Hauer and B. Zwiebach, hep-th/9801205].

[28] I. Kishimoto and N. Sasakura, hep-th/9712180.

[29] O. Bergman and A. Fayyazuddin, hep-th/9802033].

[30] Y.Imamura, hep-th/9802189.

[31] A. Mikhailov, N. Nekrasov and S. Sethi, hep-th/9803142.

[32] K. Dasgupta and S. Mukhi, Nucl. Phys. B465 (1996) 399 hep-th/9512196.

[33] E. Witten, Nucl. Phys. B463 (1996) 383 [hep-th/9512219. 
[34] A. Strominger, Phys. Lett. B383 (1996) 44 hep-th/9512059].

[35] P. Townsend, Phys. Lett. B373 (1996) 68 [hep-th/9512062.

[36] D. Kutasov, Phys. Lett. B383 (1996) 48 hep-th/9512145.

[37] A. Sen, Nucl. Phys. B474 (1996) 361 [hep-th/9604070].

[38] M. Green and M. Gutperle, Phys. Lett. B377 (1996) 28 [hep-th/9602077].

[39] M. Douglas and G. Moore, hep-th/9603167.

[40] N. Seiberg and E. Witten, Nucl. Phys. B431 (1994) 484 [hep-th/9408099].

[41] A. Bilal and F. Ferrari, Nucl.Phys. B480 (1996) 589 hep-th/9605101.

[42] J. Schwarz, Phys. Lett. B360 (1995) 13 [hep-th/9508143]. 